\title{
309 - Quality of life in caregiver in a gender perspective
}

Heidi Bjørge, PhD, Associate Professor; Kari Kvaal; Ingun Ulstein, MD PhD

Research Group: Ageing, Health, and Welfare

\section{Background and aim}

People with dementia depend on a relative to be able to stay at home longer However, being a caregiver has shown to influence on their quality of life (QoL). Depression is the most common factor associated with low caregiver QoL, while relational factors have shown to be a protective factor. Less is known about how female and male caregivers approve to the caregiving demands, and thus how these demands influence their QoL. In this study, we aimed at investigating what factors influenced on QoL related to gender and how these fluctuated with time.

\section{Method}

In this longitudinal design, a total of 208 caregivers, 158 female mean age 62.5 and 50 male caregivers, mean age 67.9 were assessed for Quality of Life-Alzheimer Disease (QoL). In addition, depression, family relationship and caregivers' burden of care were assessed. Linear regression analyses were applied to explore the bivariate and multivariate associations between the dependent variable QoL and potential explanatory variables.

The same questionnaires were completed at one-year follow-up, and the results from the remaining 176 caregivers were compared with their baseline scorings.

\section{Results}

Caregivers' depressive feelings were the strongest predictors of caregivers' QoL. What differed in the explained perceived QoL was feeling of social isolation in female caregivers, and male caregivers' perception of their care receivers' emotionally overinvolved attitude towards them. Additionally, female caregivers expressed a higher burden and cared for a relative more affected by their dementia. After one year, their QoL declined, more for male than female, while their level of depressive feelings, distress and level of EOI remained unchanged.

\section{Conclusion}

The study revealed that except for depressive feelings, female and male caregivers faced their caring experience, differently. The implication of these findings is that gender must be considered when planning for interventions. While female caregivers might need more time for leisure activities, male caregivers need ways to deal with their emotional relationship with their care receivers. Treatment of their depressive feelings seems to be crucial. They would also need approaches specifically targeting the different stages as dementia develop. 